\title{
Embedded soliton dynamics in the asymmetric array of Josephson junctions
}

\author{
Ivan O. Starodub and Yaroslav Zolotaryuk \\ Bogolyubov Institute for Theoretical Physics, National Academy of Sciences of Ukraine \\ $14 b$ vul. Metrologichna, Kyiv 03680, Ukraine \\ E-mail: starodub@bitp.kiev.ua; yzolo@bitp.kiev.ua
}

Received July 29, 2016 published online April 25, 2017

\begin{abstract}
The dc-biased annular array of three-junction asymmetric superconducting quantum interference devices (SQUIDs) is investigated. The existence of embedded solitons (solitons that exist despite the resonance with the linear waves) is demonstrated both in the unbiased Hamiltonian limit and in the dc-biased and damped case on the current-voltage characteristics (CVCs) of the array. The existence diagram on the parameter plane is constructed. The signatures of the embedded solitons manifest themselves as inaccessible voltage intervals on the CVCs. The upper boundary of these intervals is proportional to the embedded soliton velocity.

PACS: $05.45 . Y v$ Solitons;

63.20.Ry Anharmonic lattice modes;

05.45.-a Nonlinear dynamics and chaos;

03.75.Lm Tunneling, Josephson effect, Bose-Einstein condensates in periodic potentials, solitons, vortices, and topological excitations.
\end{abstract}

Keywords: superconducting quantum interference devices, current-voltage characteristics (CVCs), Josephson junctions.

\section{Introduction}

Nonlinear wave phenomena are ubiquitous in the Josephson junctions (JJs) [1,2]. It is a well established fact that such excitations as breathers [3] and topological solitons [4] can occur in systems that are based on the Josephson effect. This article will be focused on topological solitons or, as they are known in physics of JJs, fluxons. The origin of the term comes from the fact that a topological soliton in the spatially extended Josephson system (either a long Josephson junction or an array of small Josephson junctions) carries the magnetic flux quantum, $\pm \Phi_{0}= \pm(\pi \hbar / e)$. Due to their remarkable structural and dynamical stability, fluxons are widely discussed as qubits [5] or as elements in the read-out process from qubits [6]. Relativistic time dilation in the array of three-junction SQUIDs has been studied in Ref. 7.

In JJ arrays the soliton (fluxon) mobility is generally obstructed due to the discreteness of the media [8,9]. As a result, a fluxon couples with the small-amplitude wave. If the inductive coupling between the junctions is small, the free fluxon motion becomes impossible. However, the possibility of radiationless propagation of solitary waves in discrete media for selected values of velocity has been reported in the number of papers [10-20]. Also, free propagation of the bound states of several discrete topological solitons has been reported by Peyrard and Kruskal more than 30 years ago [8]. This unexpected soliton mobility is a part of the wider nonlinear phenomenon, known as embedded solitons. Embedded solitons are spatially localized solitary waves that exist for isolated parameter values (this parameter can be the soliton velocity) despite the apparent resonance with the linear waves of the system [21]. Apart from the discrete media, they also exist in various continuous systems like the sine-Gordon [22] and the double sineGordon equations [23].

The array of three-junction SQUIDs, introduced in [7] is governed by the discrete double sine-Gordon equation. In this article we demonstrate the existence of embedded solitons in this equation, study their properties in detail and show how they manifest themselves in the current-voltage characteristics of the array.

\section{Main results}

In this Section we present the model and report the main results.

\subsection{Model and equations of motion}

We investigate the array of JJs, arranged in such a way that the arms with one and two junctions alternate. The part of this array is shown schematically in Fig. 1. 


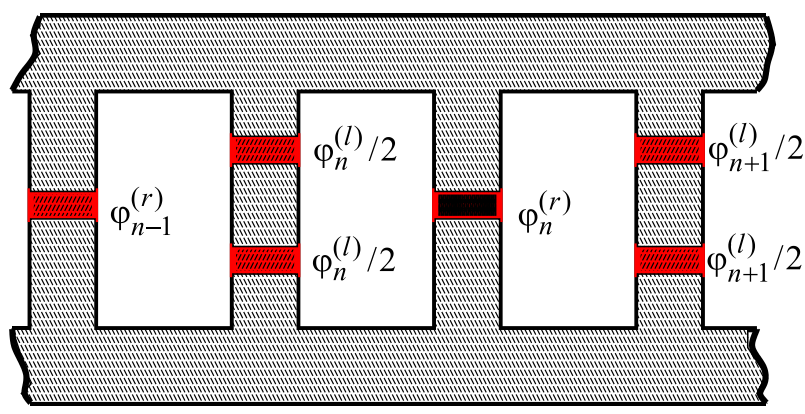

Fig. 1. (Color online) Schematic view of the SQUID array. Gray colour stands for the superconducting electrodes and the insulating barriers are marked by red. Details of the full equivalent scheme can be found in Ref. 7 .

Thus, the elementary cell of such an array is an asymmetric SQUID with two junctions in its left arm and one junction in its right arm. The elementary cell of the array is obviously asymmetric, but the whole array is not. The dynamics of each junction is described by the Josephson phases, $\varphi_{n}^{(l, r)}(t)$. They are the phase difference between the phases of the wave-functions of the superconductors on the different sides of the junction. The superscripts $l$ and $r$ stand for the left and right arms of the SQUID, respectively. The array is studied under the resistively and capacitively shunted junction-model (RCSJ-model) [1]. Based on this model and with the help of the Kirchhoff laws and the Josephson equations the equations of motion of such an array have been derived in Ref. 7. Under the assumption of the small loop size and the single phase difference $\varphi_{n}=\varphi_{n}^{(l)}=\varphi_{n}^{(r)}$ the final dimensionless equations of motion read

$$
\begin{gathered}
\ddot{\varphi}_{n}-\kappa\left(\varphi_{n+1}-2 \varphi_{n}+\varphi_{n-1}\right)+ \\
+\frac{2}{1+2 \eta}\left(\eta \sin \varphi_{n}+\sin \frac{\varphi_{n}}{2}\right)+\alpha \dot{\varphi}_{n}=\gamma \\
n=1,2, \ldots, N .
\end{gathered}
$$

This is the discrete double sine-Gordon equation (DDbSG). The current model, according to [24], takes into account only the self-inductance, while the mutual inductances of the SQUIDs are neglected. The dimensionless parameters are defined as follows

$$
\begin{gathered}
\alpha=\frac{1}{R C \omega_{J}}, \kappa=\frac{\Phi_{0}}{2 \pi L_{J} I_{C}}, \eta=\frac{I_{c}^{(r)}}{I_{c}^{(l)}}, \\
C=C_{l}+\frac{C_{r}}{2}, \frac{1}{R}=\frac{1}{R_{r}}+\frac{1}{2 R_{l}}, \quad I_{C}=I_{c}^{(r)}+\frac{I_{c}^{(l)}}{2} .
\end{gathered}
$$

Here $\omega_{J}=\sqrt{2 e I_{C} /(C \hbar)}$ is the Josephson plasma frequency and the dimensionless time in Eq. (1) is normalized in the units of $\omega_{J}^{-1}, \alpha$ is the dissipation parameter, $\Phi_{0}$ is the magnetic flux quantum, $L_{J}$ is the elementary cell induct- ance and $\gamma$ is the dimensionless external bias current, normalized to $I_{c}$. Next, $R_{r, l}, C_{r, l}$ and $I_{c}^{(r, l)}$ are, respectively, the resistance, capacitance and critical current of the right or the left junction (marked by the sub(super)script «r» or «l»). The parameter $\eta$ measures the asymmetry of the SQUID and is the ratio of the critical currents of the right and left junctions of the SQUID. The discreteness parameter $\kappa$ is responsible for the inductive coupling between the cells.

The circular array is to be considered, thus, the boundary conditions read $\varphi_{n}=\varphi_{n+N}+4 \pi Q$, where $Q$ is the total topological charge, i.e., the total number of fluxons and antifluxons trapped in the ring. In this article we restrict ourselves only to the case of one fluxon in the array, hence $Q=1$.

\subsection{Soliton mobility in the hamiltonian limit}

It is instructive to consider the DDbSG equation in the Hamiltonian (dissipationless) limit $\alpha=0$ and in the absence of the dc bias $\gamma=0$. In this case Eq. (1) takes the following form:

$$
\begin{gathered}
\ddot{\varphi}_{n}-\kappa\left(\varphi_{n+1}-2 \varphi_{n}+\varphi_{n-1}\right)=-V^{\prime}\left(\varphi_{n}\right), \\
V(\varphi)=\frac{2}{1+2 \eta}\left[\eta(1-\cos \varphi)+2\left(1-\cos \frac{\varphi}{2}\right)\right] .
\end{gathered}
$$

The periodic on-site potential $V(\varphi)=V(\varphi+4 \pi)$ has one minimum within the interval $\varphi \in[0,4 \pi]$ if $0 \leq \eta<1 / 2$. The height of the potential barrier that separates two minima at $\varphi=0$ and $\varphi=4 \pi$ is maximal if $\eta=0$ and decreases if $\eta$ is increased. An additional local minimum appears at $\varphi=2 \pi$ if $\eta>1 / 2$. It remains local for any $1 / 2 \leq \eta<\infty$, i.e., $V(2 \pi)>V(0)=0$.

The continuum version of Eq. (3) can be achieved when $\kappa \rightarrow \infty$. The continuous double sine-Gordon equation has a large number of applications [25], including the long Josephson junctions with the second harmonic in the currentphase relation [26].

In this subsection we focus on the existence of the travelling-wave topological solitons that propagate with the constant shape and velocity. They must satisfy the following condition: $\varphi_{n}(t)=\varphi(n-v t) \equiv \varphi(z)$. Thus, for these solutions Eq. (3) can be rewritten as

$v^{2} \varphi^{\prime \prime}(z)-\kappa[\varphi(z+1)+\varphi(z-1)-2 \varphi(z)]+V^{\prime}[\varphi(z)]=0$.

This is a differential-difference ODE with the delay and advance terms. Analytical solution of such equations is not possible. In some special cases it is possible to find its isolated travelling-wave solutions $[10,11]$. Therefore we employ the numerical technique, developed in Refs. 27-29. The solution is assumed to be periodic with rather large period $L$ that exceeds significantly all characteristic sizes of the system and is sought in the form of the Fourier series 


$$
u(z) \simeq u_{0}(z)+\sum_{n=0}^{k} c_{n} \sin \left(\frac{2 \pi n z}{L}\right),
$$

where the initial approximation $u_{0}(z)$ is used in order to enhance the initial guess and to enforce the appropriate boundary conditions. It should be noted that the elementary topological soliton in the DDbSG equation connects the ground states $\varphi=0$ and $\varphi=4 \pi$. Coefficients $c_{n}$ are to be determined. The integer part of $L$ will effectively play the role of the chain length in the original system (1). After substituting the ansatz (6) into Eq. (5) and breaking the interval $z \in[0, L / 2]$ into $k$ collocation points we obtain $k$ nonlinear algebraic equations for the coefficients $\left\{c_{n}\right\}_{n=1}^{k}$. These equations can be solved with the Newton-Raphson method or any other appropriate scheme.

Currently it is a well-established fact [10-14,18-20] that for the discrete Klein-Gordon equations of the type (3), the continuous family of moving solitons turns into the discrete finite set of monotonic $\left(\lim _{|n| \rightarrow \infty} \varphi_{n} \rightarrow\right.$ const) travelling kink solutions with the velocities $v=\left\{v_{0} \equiv 0, v_{1}, v_{2} \ldots, v_{k}\right\}$. Further on, all velocities that satisfy $v_{n} \neq 0$ will be called sliding velocities since the kink slides along the lattice with these velocities without any radiation. In the DSG equation there are only non-mobile $\left(v_{0}=0\right)$ monotonic solitons and there is no sliding velocities for solitons with topological charges $Q= \pm 1$. There exist embedded solitons for higher topological charges, $Q= \pm 2, \pm 3, \ldots$, which are, in fact, bound states of several solitons or «soliton complexes» $[8,23]$ that appear due to destructive interference between the elementary solitons. In general, everywhere away from the sliding velocities, i.e., if $v \neq v_{n}$, the moving kinks are non-monotonic, have oscillating asymptotic tails and are often referred to as nanopterons.

Appearance of nanopterons is natural and its origin is the resonance that happens due to the fact $[8,30]$ that any soliton which moves in the lattice governed by Eq. (3) with velocity $|v|<1$ will always excite a small-amplitude wave that moves with the same phase velocity. In other words, the equation

$$
v q=\omega_{L}(q)=\sqrt{1+4 \kappa \sin ^{2} \frac{q}{2}},
$$

where $\omega_{L}(q)$ is the spectrum of the small-amplitude waves of Eq. (3), always has at least one real root if $|v|<1$.

These solutions can be called discrete embedded solitons, similarly to their continuous counterparts [21,22] because they exist despite the resonance with the smallamplitude waves and are «embedded» in the continuous family of nanopterons. The spatial monotonicity of these solutions, their localized nature, and, as a result their finite energy also contribute to their importance.

Our numerical investigation of the DDbSG equation has shown that embedded solitons can exist there as well. In Fig. 2 we plot the dependence of the nanopteron amplitude tail as a function of the velocity. We have fixed the cou-

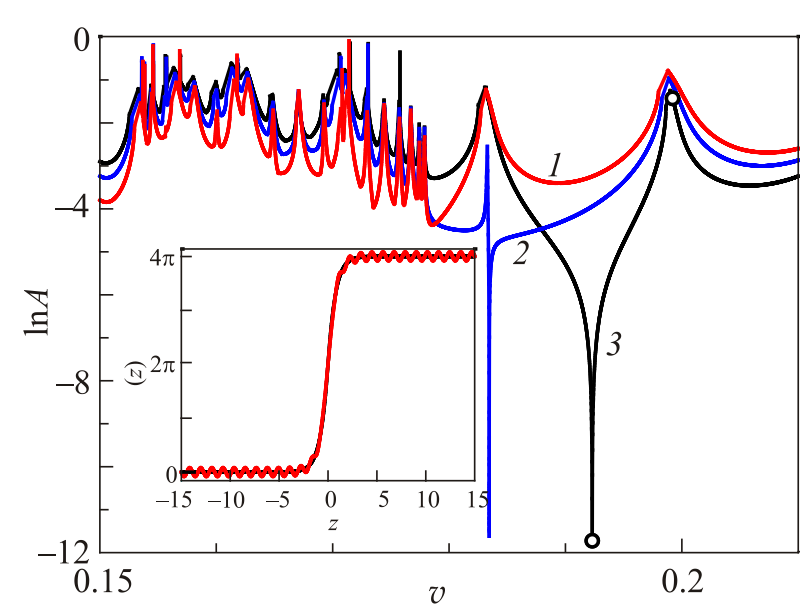

Fig. 2. (Color online) Dependence of the tail amplitude $A$ on the moving soliton velocity for $L=30, \kappa=0.4, \eta=0.26$ (curve 1 , red), $\eta=0.27$ (curve 2, blue) and $\eta=0.28$ (curve 3 , black). The inset shows the soliton profile for $v=0.192302$ (red) and $v=0.1992$ (black). Respective position of these solutions on the $A(s)$ curve are marked by $(O)$.

pling constant to $\kappa=0.4$ and varied the asymmetry parameter $\eta$. One can observe that a slight change of $\eta$ from $\eta=0.26$ (red curve) to $\eta=0.27$ (blue curve) can bring a significant change, namely a selected value of velocity for which $A \rightarrow 0$ in an extremely sharp fashion (note the logarithmic scale).

This is the sliding velocity mentioned above. The solution that corresponds to this velocity is spatially localized embedded soliton with the monotonic asymptotical behavior (see inset of Fig. 2). Away from this velocity we find nanopterons or bound states of solitons and plane waves. An example of a nanopteron is also plotted in the inset of Fig. 2. For the sake of clarity only a small segment of velocities was considered on this figure. No other sliding velocities have been found outside of this segment.

From Fig. 2 one can notice that the dependences $A(v)$ have sharp maxima. In fact, they are not maxima but singularities that appear because the numerical scheme is not able to find a solution with the appropriate wavelength. This is a consequence of the boundary conditions and these singularities depend on the length $L$. For the velocity that lies between the two adjacent singularities the nanopteron solution has a certain fixed number of small-amplitude wavelength that fit into the interval $[-L / 2, L / 2]$. The wavelength equals $2 \pi / q$ where the wavenumber $q$ is the root of Eq. (7). In the case of several roots there exist several nanopteron solutions with different wavelengths. As one changes $v$, passes the singularity and enters the next interval the number of fitted wavelengths changes.

If one of the pair of parameters, $\kappa$ or $\eta$ is fixed and another one is varied, the critical parameter value of the variable parameter can be found, below which there is no sliding velocities. This is demonstrated in Fig. 3(a), where the 

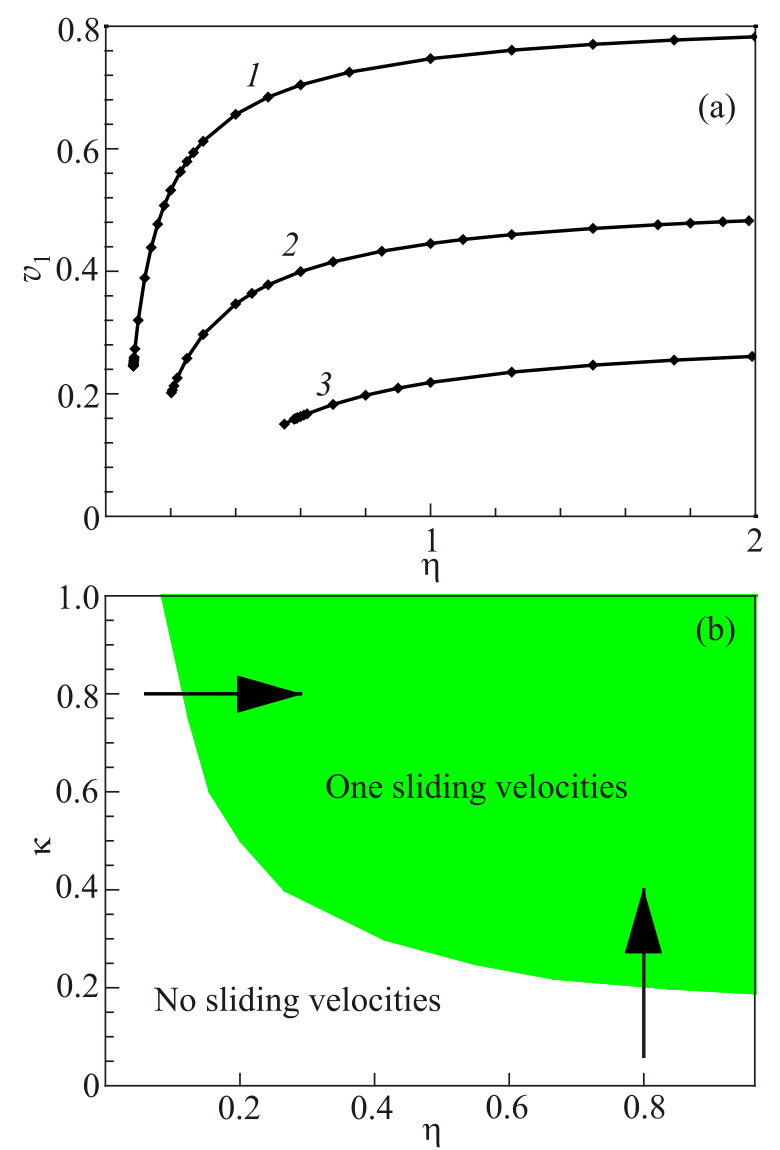

Fig. 3. (Color online) (a) The value of the sliding velocity $v_{1}$ as a function of the asymmetry parameter $\eta$ for different values of the coupling constant: $\kappa=1$ (curve 1), $\kappa=0.5$ (curve 2) and $\kappa=0.25$ (curve 3). (b) The existence diagram on the parameter plane $(\kappa, \eta)$. One sliding velocity exists in the green area while there is no sliding velocities in the white area. All results were obtained for $L=30$.

dependence of the sliding velocity $v_{1}$ is plotted as a function of the asymmetry parameter $\eta$ for the different values of $\kappa$. Interestingly, the embedded solitons can exist for the strongly discrete arrays. The existence diagram on the plane $(\kappa, \eta)$, presented in Fig. 3(b), generalizes the above result.

It is possible to draw a line on this plane that separates the area without sliding velocities (and, consequently, with no embedded solitons) from the area where one sliding velocity exists. For the DDbSG equation we were able to find only one sliding velocity. In some models there can be more of them $[12,13]$.

\subsection{Current-voltage characteristics}

Now we focus on the more realistic situation when the array is dissipative $(\alpha>0)$ and dc-biased $(\gamma \neq 0)$. The current-voltage characteristics (CVCs) provide the necessary information about the JJ array dynamics and can be measured experimentally. We have varied the bias and computed the average voltage drop

$$
\bar{V}=\frac{1}{N} \sum_{n=1}^{N} \lim _{t \rightarrow \infty} \frac{1}{t} \int_{0}^{t} \dot{\varphi}_{n}\left(t^{\prime}\right) d t^{\prime} .
$$

If there is a fluxon in the array that moves with the constant velocity $v$, the average voltage drop will be $\bar{V}=4 \pi v / N$.

The numerically obtained CVCs are given in Fig. 4. The coupling constant and the array size were fixed to $\kappa=0.5$ and $N=30$, respectively, while we have changed the asymmetry parameter $\eta$ and the damping constant $\alpha$. It appears that there exists a significant difference between the CVCs if the system supports embedded soliton and when it does not. The numerically computed CVCs are shown by the different markers while the solid line corresponds to the CVC that was obtained from the continuum approximation [36]:

$$
\begin{gathered}
\bar{V}_{c}=\sqrt{\kappa} \frac{4 \pi v_{\infty}}{N}=\sqrt{\kappa} \frac{4 \pi}{N}\left[1+\Phi^{2}(\eta)\left(\frac{4 \alpha}{\pi \gamma}\right)^{2}\right]^{-1 / 2}, \\
\Phi(\eta)=1+\frac{1}{\sqrt{2 \eta(2 \eta+1)}} \operatorname{arctanh} \sqrt{\frac{2 \eta}{1+2 \eta}}
\end{gathered}
$$

This formula is very similar to the well-known McLaughlin-Scott result for the fluxon motion in the ordinary long $\mathrm{JJ}$ [4]. It is reduced to the McLaughlin-Scott formula in the both limits: $\eta \rightarrow 0$ and $\eta \rightarrow \infty$. The continuum version is plotted here mainly as a reference point, but appears to work surprisingly well even in this strongly discrete case.

First of all we focus on the principal differences between fluxon mobility in discrete and continuous media. The fluxon velocity in the continuous $\mathrm{JJ}$ is defined uniquely by the ratio $\gamma / \alpha$ and, as one can see in the CVCs in Fig. 4, the respective characteristics are continuous functions that pass through the origin. There are two main differences in the discrete case: (i) the CVC does not pass through the origin; (ii) one continuous branch is replaced by the set of roughly parallel branches. The first difference is the natural consequence of the discreteness, because some finite bias is always needed to overcome the lattice pinning. The second difference is caused by the boundary conditions and by the fluxon coupling with the linear modes [30-34]. While moving along the array, the fluxon excites the linear modes and forms a bound state that propagates with the same velocity. The wavelength of the linear mode is given by Eq. (7). Because of the periodic boundary conditions the phase locking in the array would occur if the finite number of the Josephson phase oscillations fits into one cycle of the fluxon journey along the array. The phase locking means that the system settles on the periodic attractor that corresponds to the fluxon restoring its shape and position completely after circumventing the array. In other words, a certain number of the linear modes wavelength should be fitted in the array and the different branches of the CVC in Fig. 4 correspond to the different number of these wavelengths. 


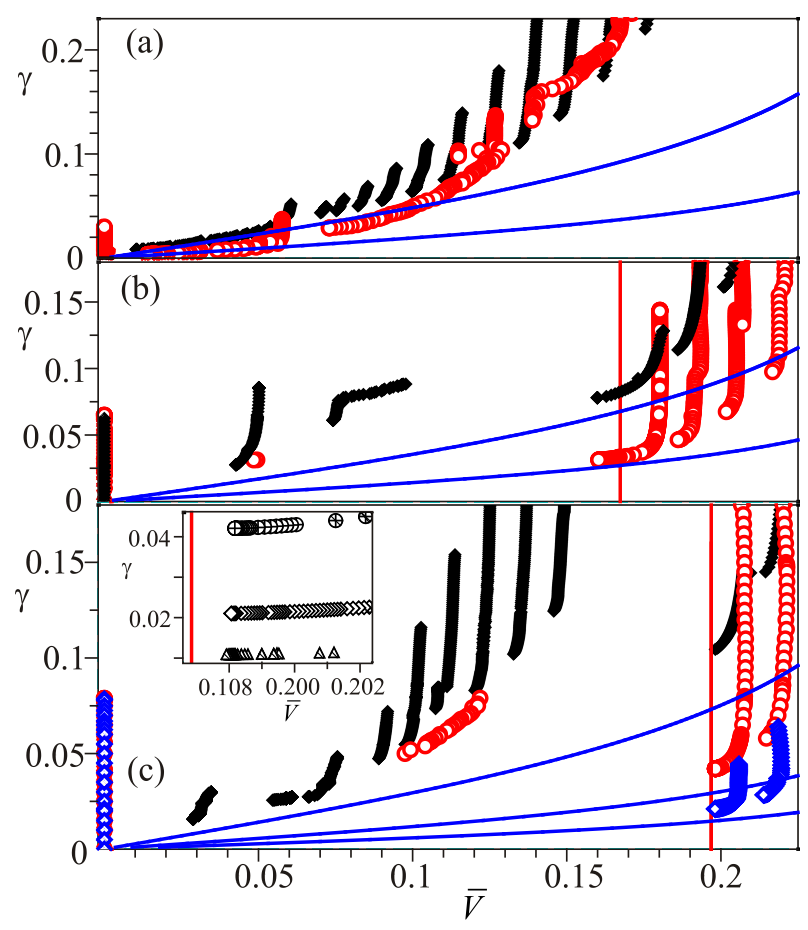

Fig. 4. (Colour online) Current-voltage curves for $\kappa=0.5, N=30$, $\alpha=0.05$ (black $\diamond$ ), $\alpha=0.02$ (red $\bigcirc$ ), $\alpha=0.01$ (blue $\diamond$ ) and $\eta=0.1$ (a), $\eta=0.6$ (b) and $\eta=1.5$ (c). The blue solid lines correspond to the respective CVC in the continuum limit. The red vertical lines in (b), (c) are given by $4 \pi v_{1} / N$, where $v_{1}$ is the sliding velocity for the respective value of $\eta$. The inset in the panel (c) shows the details of CVCs in the neighbourhood of the sliding velocity for $\alpha=0.02(\oplus), \alpha=0.01(\diamond)$ and $\alpha=0.005(\triangle)$.

Now we discuss the signatures of the embedded solitons on the CVCs. In Fig. 4(a) the case of $\eta=0.1$ is considered, and, according to the previous subsection, there is no embedded solitons in the hamiltonian limit for this value of the asymmetry parameter. On contrary, for $\eta=0.6$ (Fig. 4(b)) and for $\eta=1.5$ (Fig. 4(c)) there exist one embedded soliton with the sliding velocity $v_{1}=0.399493$ and $v_{1}=0.469944$, respectively. For details see Fig. 3. In the first case the CVC branches are distributed almost uniformly. In the second and third cases one can easily spot the significant gap $V_{I V I}^{-} \leq V \leq V_{I V I}^{+}$which will be called inaccessible voltage interval (IVI). This IVI appears more pronounced as $\eta$ increases or when $\alpha$ is decreased. Moreover, if $\alpha$ is decreased significantly, its lower boundary, equals zero. The upper boundary, $V_{I V I}^{+}$tends to the value $4 \pi v_{1} / N$, which is the voltage drop produced by the embedded soliton (marked by the red vertical line). We have reduced $\alpha$ and computed the detuning $v=\left|4 \pi v_{1} / N-V_{I V I}^{+}\right|$. For the lowest value of dissipation which we have used in our numerical analysis, $\alpha=0.005$, the detuning value reached $v \sim 10^{-3}$.
From these data one can make the following conclusion. If in the hamiltonian limit the JJ array supports the embedded soliton that moves with velocity $v$, in the weakly driven and weakly dissipative array this will be the dominant attractor of the system. Thus, the average voltage drop in this limit is defined by the sliding velocity and is far from zero. If the system does not support embedded solitons, its behavior in the limit $\alpha \rightarrow 0, \gamma \rightarrow 0$ is different: the CVC ends up close to the origin, as shown in Fig. 4(a). However, it does not pass the origin due to discreteness.

The small-wave radiation intensity

$$
I(\Omega)=\left|\int_{-\infty}^{+\infty} \dot{\varphi}_{N / 2}(t) \mathrm{e}^{-i \Omega t} d t\right|^{2},
$$

that appears during the fluxon motion around the array is shown in Fig. 5. Here the fast Fourier transform (FFT) is taken for the $N / 2$ th junction of the array. The time interval $\left[t_{0}, t_{\text {fin }}\right]$ for FFT was chosen in such a way that the fluxon motion did not contribute to the power spectrum. We have focused on the array parameters that correspond to Fig. 4(c) and for the dissipation value $\alpha=0.02$. Three points on the CVC branch that ends at $V_{I V I}^{+} \sim 0.2$ were chosen for the power spectrum analysis. Since the fluxon velocity is close to the sliding velocity $v_{1}=0.469944$, the time for the fluxon to travel around the array is $N / v_{1} \sim 60$. Therefore, we have taken $t_{\text {fin }}-t_{0}=50$.

We take the CVC branch that is adjacent to the upper bond of the IVI. If the bias is decreased we move down the branch, and the amount of the radiated energy decreases significantly (note the log scale). The peak of the emitted waves lies within the linear band (7).

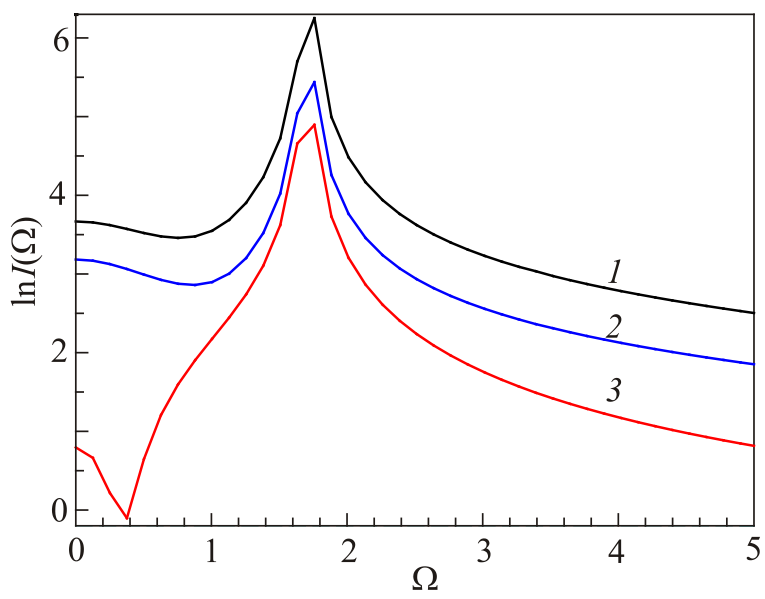

Fig. 5. (Color online) Radiation intensity of the plane waves in the JJ array with the parameters that correspond to Fig. 4(c) and with $\alpha=0.02$. The respective values of the dc bias and voltage are: $\gamma=0.05, \bar{V} \simeq 0.205$ (curve 1, black), $\gamma=0.044, \bar{V} \simeq 0.201$ (curve 2, blue) and $\gamma=0.0423, \bar{V} \simeq 0.199$ (curve 3, red). 


\section{Conclusions}

In this paper we have demonstrated that the array of asymmetric three-junction SQUIDs, which is described by the discrete double sine-Gordon equation, supports embedded solitons. This equation is also used to describe the parallel array of the superconductor-ferromagnet-superconductor (SFS) or superconductor-ferromagnet-insulatorsuperconductor (SFIS) junctions, that are governed by the biharmonic current-phase relation

$$
I_{c}(\varphi)=I_{c, 1} \sin \varphi+I_{c, 2} \sin 2 \varphi
$$

Embedded solitons are spatially localized excitations that exist despite the resonance with the small-amplitude waves of the underlying system. They exist for the isolated set of velocities. The important parameter of the model is the ratio $\eta$ of the critical currents of the left and right arms of the SQUID, which we call the asymmetry parameter. These embedded solitons manifest themselves on the current-voltage characteristics of the JJ array as inaccessible voltage intervals (IVI), i.e., the forbiden values of voltage. The upper bound of such an IVI tends to $4 \pi v / N$ as $\alpha \rightarrow 0$, where $v$ is the embedded soliton velocity in the hamiltonian limit. The lower bound of the IVI tends to zero if the asymmetry parameter is large enough and dissipation is small enough.

This phenomenon should not be confused with the series of results on the models with the non-zero Peiers-Nabarro barrier [16,39,40]. For the discrete embedded solitons the Peierls-Nabarro barrier is non-zero [12,13], however, this does not prevent the free soliton propagation.

The symmetric SQUID array or, equivalently, the array of parallel shunted small Josephson junctions [35] is described by the DSG equation. It has been demonstrated both theoretically $[8,12,14,35]$ and experimentally [37] that the radiationless motion of the coupled state of several solitons is possible for the selected set of their velocities. This phenomenon has been treated analytically in the quasi-continuum approximation in Refs. 22, 23, but it takes place even in the sufficiently discrete array $(\kappa<1)$ as well. In the limit $\eta \rightarrow \infty$ the double SG equation becomes the ordinary SG equation with the period $2 \pi$, thus, the abovementioned result of the bound state of two $2 \pi$ solitons is the special case of the kink mobility of the DDbSG equation in the limit $\eta \rightarrow \infty$.

\section{Acknowledgements}

The authors acknowledge the financial support from the National Academy of Sciences of Ukraine (NAS of Ukraine) under the Project No. 0112 U000053 and from the Project 1/30-2015 "Dynamics and topological structures in Bose-Einstein condensates of ultracold gases" of the Kyiv National University Branch Target Training Program at the NAS of Ukraine.
1. A. Barone and G. Paterno, Physics and Applications of the Josephson Effect, Wiley, New York (1982).

2. A.V. Ustinov, Physica D 123, 315 (1998).

3. E. Trías, J.J. Mazo, and T.P. Orlando, Phys. Rev. Lett. 84, 741 (2000); P. Binder, D. Abraimov, A. V. Ustinov, S. Flach and Y. Zolotaryuk, Phys. Rev. Lett. 84, 745 (2000).

4. D.W. McLaughlin and A.C. Scott, Phys. Rev. A 18, 1652 (1978).

5. D.V. Averin, K. Rabenstein, and V.K. Semenov, Phys. Rev. B 73, 094504 (2006).

6. A. Fedorov, A. Shnirman, G. Schon, and A. KidiyarovaShevchenko, Phys. Rev. B 75, 224504 (2007).

7. M. Nishida, T. Kanayama, T. Nakajo, T. Fujii, and N. Hatakenaka, Physica C 470, 832 (2010).

8. M. Peyrard and M.D. Kruskal, Physica D 14, 88 (1984).

9. O.M. Braun and Y.S. Kivshar, Phys. Rep. 306, 2 (1998).

10. S. Flach, Y. Zolotaryuk, and K. Kladko, Phys. Rev. E 59, 6105 (1999).

11. V.H. Schmidt, Phys. Rev. B 20, 4397 (1979).

12. A.V. Savin, Y. Zolotaryuk, and J.C. Eilbeck, Physica D 138, 265 (2000).

13. V.M. Karpan, Y. Zolotaryuk, P.L. Christiansen, and A.V. Zolotaryuk, Phys. Rev. E 66, 066603 (2002).

14. A. Aigner, A. Champneys, and V. Rothos, Physica D 186, 148 (2003).

15. Y. Zolotaryuk, J.C. Eilbeck, and A.V. Savin, Physica D 108, 81 (1997).

16. I.V. Barashenkov, O.F. Oxtoby, and D.E. Pelinovsky, Phys. Rev. E 72, 035602(R) (2005).

17. B.A. Malomed, J. Fujioka, A. Espinosa-Cerón, R.F. Rodríguez, and S. González, Chaos 16, 013112 (2006).

18. O. Oxtoby, D.E. Pelinovsky, and I.V. Barashenkov, Nonlinearity 19, 217 (2006).

19. S.V. Dmitriev, A. Khare, P.G. Kevrekidis, A. Saxena, and L. Hadzievski, Phys. Rev. E 77, 056603 (2008).

20. G.L. Alfimov, E.V. Medvedeva, and D.E. Pelinovsky, Phys. Rev. Lett. 112, 054103 (2014).

21. A. Champneys, B. Malomed, J. Yang, and D. Kaup, Physica D 152-153, 340 (2001).

22. A. Champneys and Y.S. Kivshar, Phys. Rev. E 61, 2551 (2000).

23. M.M. Bogdan, A. Kosevich, and G.A. Maugin, Wave Motion 34, 1 (2001).

24. R.D. Bock, J.R. Phillips, H.S.J. van der Zant, and T.P. Orlando, Phys. Rev. B 49, 10009 (1994).

25. C.A. Condat, R.A. Guyer, and M.D. Miller, Phys. Rev. B 27, 474 (1983); D.K. Campbell, M. Peyrard, and P. Sodano, Physica D 19, 165 (1986).

26. E. Goldobin, D. Koelle, R. Kleiner, and A. Buzdin, Phys. Rev. B 76, 224523 (2007).

27. D. Hochstrasser, F. Mertens, and H. Büttner, Physica D: Nonlinear Phenomena 35, 259 (1989).

28. J.C. Eilbeck and R. Flesch, Phys. Lett. A 149, 200 (1990).

29. D. Duncan, J. Eilbeck, H. Feddersen, and J. Wattis, Physica D 68, 1 (1993). 
30. A.V. Ustinov, M. Cirillo, and B.A. Malomed, Phys. Rev. B 47, 8357 (1993).

31. S. Watanabe, H.S.J. van der Zant, S.H. Strogatz, and T.P. Orlando, Physica D 97, 429 (1996).

32. O.M. Braun, B. Hu, and A. Zeltser, Phys. Rev. E 62, 4235 (2000).

33. A.V. Ustinov, M. Cirillo, and B.A. Malomed, Phys. Lett. A 183, 383 (1993).

34. A.V. Ustinov, M. Cirillo, Britt H. Larsen, V.A. Oboznov, P. Carelli, and G. Rotoli Phys. Rev. B 51, 3081 (1995).
35. A.V. Ustinov, B.A. Malomed, and S. Sakai, Phys. Rev. B 57, 11691 (1998).

36. Y. Zolotaryuk and I.O. Starodub, Phys. Rev. E 91, 013202 (2015).

37. J. Pfeiffer, M. Schuster, A.A. Abdumalikov, and A.V. Ustinov, Phys. Rev. Lett. 96, 034103(4) (2006).

38. A.A. Golubov, M.Y. Kupriyanov, and E. Ilichev, Rev. Mod. Phys. 76, 411 (2004).

39. M. Speight and Y. Zolotaryuk, Nonlinearity 19, 1365 (2006).

40. S.V. Dmitriev, P.G. Kevrekidis, N. Yoshikawa, and D.J. Frantzeskakis, Phys. Rev. E 74, 056609 (2006). 